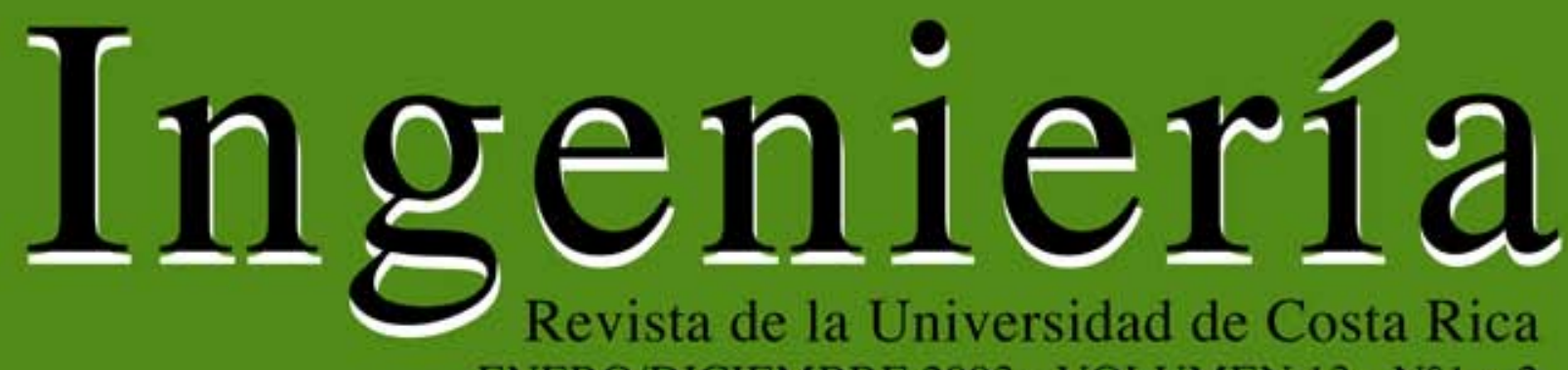
ENERO/DICIEMBRE 2003 - VOLUMEN 13 - N¹ y 2

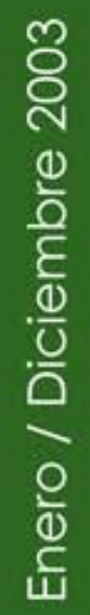

$\frac{m}{5}$
$\frac{c}{d}$
$\frac{5}{5}$
$\frac{\partial}{\circ}$

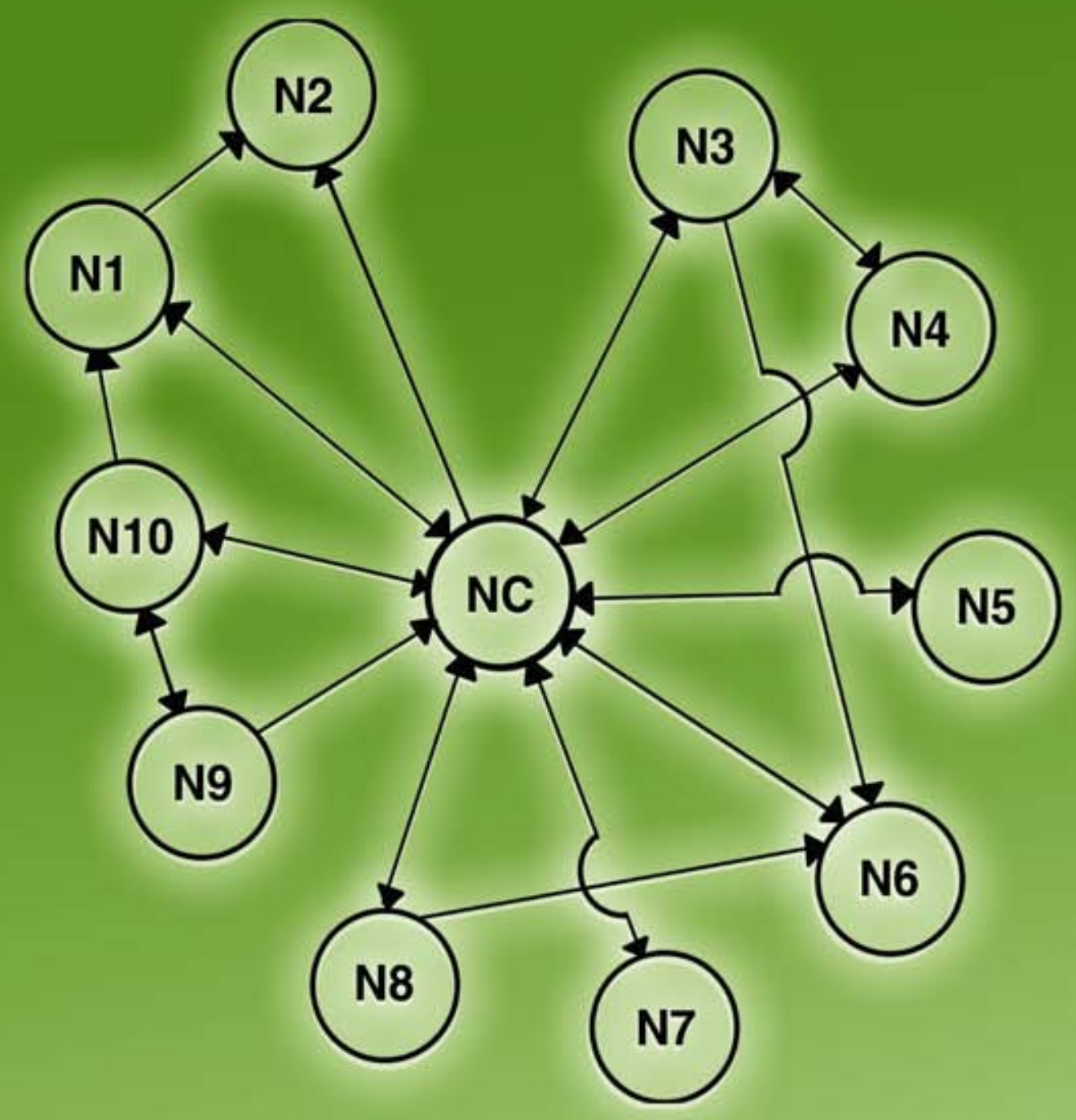

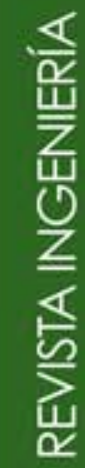




\title{
MÉTODOS DE SINTONIZACIÓN DE CONTROLADORES PID QUE OPERAN COMO SERVOMECANISMOS
}

\author{
Víctor M. Alfaro Ruiz
}

\section{Resumen}

Se presentan varios de los métodos de sintonización de controladores PID para lazos de control que funcionan como servomecanismos. Mediante pruebas de simulación digital, se compara el desempeño de los diferentes métodos y se determina la bondad de los mismos con base en criterios de comportamiento y robustez. Finalmente, se dan recomendaciones sobre la utilización de los métodos probados.

Palabras clave: controladores PID, sintonización, servomecanismos.

\begin{abstract}
Several methods for tuning servomechanism PID controllers are presented. The behavior and goodness of the tuning methods are compared by digital simulation using several performance and robustness criteria. Recommendations for the use of the tested tuning methods are given based on the performance requirements.
\end{abstract}

Keywords: PID controllers, tuning rules, set point changes.

\section{INTRODUCCIÓN}

La sintonización de los controladores Proporcional - Integral - Derivativo o simplemente controladores PID, consiste en la determinación del ajuste de sus parámetros $\left(K_{c}, T_{i}, T_{d}\right)$, para lograr un comportamiento del sistema de control aceptable y robusto de conformidad con algún criterio de desempeño establecido.

Para poder realizar la sintonización de los controladores, primero debe identificarse la dinámica del proceso, y a partir de ésta determinar los parámetros del controlador utilizando el método de sintonización seleccionado.

Antes de revisar algunas de las técnicas de sintonización disponibles, se establecerán los diferentes tipos de funcionamiento que pueden presentarse en un lazo de control realimentado, en el cual hay dos entradas - el valor deseado $r(t)$ y la perturbación $z(t)$ - y una salida - la señal realimentada $y(t)$-, como se muestra en la Figura 1.
$\mathrm{Si}$ se considera las variables en el dominio de la variable compleja s y se define $G_{c}(s)$ y $G_{p}(s)$ como las funciones de transferencia del controlador y de la planta respectivamente, a partir del diagrama de bloques del sistema de control de lazo cerrado, se obtiene que la señal realimentada, representación de la variable controlada, está dada por:

$y(s)=\frac{G_{C}(s) G_{p}(s)}{1+G_{C}(s) G_{p}(s)} r(s)+\frac{G_{p}(s)}{1+G_{C}(s) G_{p}(s)} z(s)$

Se debe considerar, entonces, dos posibles condiciones de operación del sistema de control, en primer lugar:

- $\quad \operatorname{Servomecanismo~}(z=0)$

$y(s)=\frac{G_{C}(s) G_{p}(s)}{1+G_{C}(s) G_{p}(s)} r(s)$

Esta condición requiere un buen seguimiento al valor deseado. En segundo lugar: 
- Regulador $(r=0)$

$y(s)=\frac{G_{p}(s)}{1+G_{c}(s) G_{p}(s)} z(s)$

en donde lo importante es la insensibilidad a las perturbaciones.

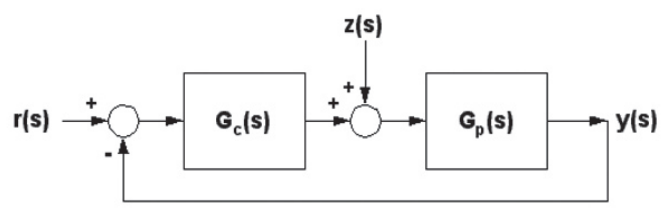

Figura 1. Sistema de control realimentado

Dado que los numeradores de las funciones de transferencia de lazo cerrado (2) y (3) son diferentes, no necesariamente un buen ajuste del controlador para operar como servomecanismo, proveerá un buen funcionamiento como regulador, razón por la cual se han desarrollado procedimientos de sintonización para ambos tipos de operación.

En la industria de procesos, la mayor parte de los controladores se utilizan para responder a un cambio en la perturbación, se requiere de una buena regulación, y no para seguir un cambio en el valor deseado, con excepción de los controladores esclavos en los sistemas de control en cascada. Si el controlador se ha sintonizado para lograr una buena respuesta en un cambio en el valor deseado, no eliminará las perturbaciones en forma efectiva, si el sistema contiene un integrador o si su constante de tiempo es grande.

Es importante, entonces, determinar los requisitos de funcionamiento del lazo de control para seleccionar el procedimiento de sintonización adecuado.

El desarrollo de los métodos de sintonización ha sido extenso desde que Ziegler y Nichols propusieron su procedimiento en 1942. Normalmente, se dividen en métodos de lazo abierto y métodos de lazo cerrado:

\section{- Métodos de lazo abierto}

El controlador si está instalado se operará en modo manual. Produciendo un cambio escalón a la salida del controlador se obtiene la curva de reacción del proceso, a partir de la cual se identifica un modelo para el mismo, usualmente de primer orden más tiempo muerto. Este modelo es la base para la determinación de los parámetros del controlador.

\section{- Métodos de lazo cerrado}

El controlador se debe operar en modo automático, y producir un cambio en el valor deseado para obtener información del comportamiento dinámico del sistema, e identificar un modelo de orden reducido para el proceso, o las características de oscilación sostenida del mismo, para utilizarla en el cálculo de los parámetros del controlador.

Se restringirá la presentación de los métodos de sintonización y de los resultados de la comparación, a aquéllos desarrollados para controladores PID que operan como servomecanismos y utilizan un modelo del proceso. En particular, se hará referencia a los siguientes métodos por ser los más empleados:

- (Rovira, Murrill y Smith, 1969)

- (Kaya y Sheib, 1988)

- (Sung et al., 1996)

- (Rivera, Morari y Stogestad, 1985)

- (Brosilow, 1992)

(O’Dwyer, 2000a) y (O’Dwyer, 2000b) resume una gran variedad de procedimientos de sintonización para controladores PI y PID más.

\section{CONTROLADORES}

Los controladores PID considerados por los autores de los métodos de sintonización presentan alguna de las siguientes funciones de transferencia (Alfaro, 1993): 
- Controlador PID-Ideal

$u(s)=K_{C}\left[1+\frac{1}{T_{i} s}+\frac{T_{S} s}{1+\tau_{f} s}\right](r(s)-y(s))$

- Controlador PID-Serie (Interactuante)

$u(s)=K_{c}^{\prime}\left[1+\frac{1}{T_{i}^{\prime} s}\right]\left[1+\frac{T_{d}^{\prime} s}{1+\tau_{f} s}\right](r(s)-y(s))$

- Controlador PID-Industrial

$u(s)=K_{C}^{\prime}\left[1+\frac{1}{T_{i}^{\prime} s}\right]\left[r(s)-\frac{T_{d}^{\prime} s}{1+\tau f s} y(s)\right]$

Si el controlador PID, que se desea sintonizar, no es del mismo tipo que el supuesto en el método de sintonización a emplear, deberá realizarse, la conversión de parámetros necesaria.

\section{MÉTODOS DE SINTONIZACIÓN}

\subsection{Modelos}

Los procedimientos de sintonización de lazo abierto utilizan un modelo de la planta, que se obtiene, generalmente, a partir de la curva de reacción del proceso, son:

- $\quad$ Primer orden más tiempo muerto

$G_{p}(s)=\frac{k_{p} e^{-t_{m} s}}{\tau s+1}$

- Polo doble más tiempo muerto

$G_{p}(s)=\frac{k_{p} e^{-t_{m} s}}{(\tau s+1)^{2}}$

- $\quad$ Segundo orden más tiempo muerto

$$
\begin{aligned}
& G_{p}(s)=\frac{k_{p} e^{-t_{m} s}}{\left(\tau_{1} s+1\right)\left(\tau_{2} s+1\right)} \\
& G_{p}(s)=\frac{k_{p} e^{-t_{m} s}}{\tau^{2} s^{2}+2 \varsigma \tau s+1}
\end{aligned}
$$

(Alfaro, 2001a) y (Alfaro, 2001b) presenta y compara algunas de las técnicas de identificación de lazo abierto y lazo cerrado más utilizadas para la obtención de estos modelos.

\subsection{Método de Rovira, Murrill y Smith}

Siguiendo el trabajo desarrollado por López para controladores PID que operan como reguladores, (Rovira et al., 1969) presentaron el primer método basado en criterios integrales para el cálculo de los parámetros del controlador que operan como servomecanismo, conocido como el método de Rovira.

Definiendo una función de costo de la siguiente forma:

$\Phi=\int_{0}^{\infty} F[e(t), t] d t$

donde $F$ es una función del error y del tiempo, se obtiene un valor que caracteriza la respuesta del sistema. Entre menor sea el valor de, mejor será el desempeño del sistema de control por lo que un desempeño óptimo se obtiene cuando sea mínimo.

Como es función de los parámetros del controlador $\left(K_{c}, T_{i}, T_{d}\right)$ el valor mínimo de se obtiene resolviendo las ecuaciones

$\frac{\partial \Phi}{\partial K_{c}}=0, \frac{\partial \Phi}{\partial T_{i}}=0, \frac{\partial \Phi}{\partial T_{d}}=0$

Los criterios de desempeño utilizados por Rovira fueron la Integral del error absoluto (IAE) y la Integral del error absoluto por el tiempo (ITAE).

La optimización de los criterios de desempeño integrales está basada en el mejor modelo de primer orden más tiempo muerto que se pueda obtener, para lazos de control funcionado como servomecanismos, con un controlador PIDIdeal. 
Las ecuaciones de sintonización son:

$K_{c} k_{p}=a\left(\frac{t_{m}}{\tau}\right)^{b}$

$\frac{T_{i}}{\tau}=\frac{1}{c+d\left(t_{m} / \tau\right)}$

$\frac{T d}{\tau}=e\left(\frac{t_{m}}{\tau}\right)^{f}$
Los valores para las constantes $a$ a $f$ para los diferentes criterios se listan en la Cuadro 1.

\subsection{Método de Kaya y Sheib}

Mientras Rovira desarrolló un método de sintonización para un controlador PID-Ideal, (Kaya y Sheib, 1985) realizaron lo mismo para controladores que denominaron PID-Clásico (PID-Serie), PID-No Interactuante (una variación del PID-Paralelo) y el PID-Industrial.

Cuadro 1. Constantes para las ecuaciones del método de Rovira

$\begin{array}{lcccccc} & \mathbf{a} & \mathbf{b} & \mathbf{c} & \mathbf{d} & \mathbf{e} & \mathbf{f} \\ \boldsymbol{I A E} & 1,086 & -0,869 & 0,740 & -0,130 & 0,348 & 0,914 \\ \boldsymbol{I} \boldsymbol{T} \boldsymbol{E} \boldsymbol{E} & 0,965 & -0,850 & 0,796 & -0,1465 & 0,308 & 0,929\end{array}$

Cuadro 2. Constantes para las ecuaciones del método de Kaya y Sheib

\section{Controlador "clásico" - Servomecanismo}

$\begin{array}{lcccccc} & \mathbf{a} & \mathbf{b} & \mathbf{c} & \mathbf{d} & \mathbf{e} & \mathbf{f} \\ \boldsymbol{I A \boldsymbol { E }} & 0,65000 & -1,04432 & 0,98950 & 0,09539 & 0,50814 & 1,08433 \\ \boldsymbol{I} \boldsymbol{T} \boldsymbol{E} \boldsymbol{E} & 1,12762 & -0,80368 & 0,99783 & 0,02860 & 0,42844 & 1,00810 \\ \boldsymbol{I S E} & 0,71959 & -1,03092 & 1,12666 & -0,18145 & 0,54568 & 0,86411\end{array}$

Controlador "no interactuante" - Servomecanismo

$\begin{array}{lcccccc} & \mathbf{a} & \mathbf{b} & \mathbf{c} & \mathbf{d} & \mathbf{e} & \mathbf{f} \\ \boldsymbol{I A E} & 1,13031 & -0,81314 & 5,75270 & -5,72410 & 0,32175 & 0,17707 \\ \boldsymbol{I} \boldsymbol{E} \boldsymbol{E} \boldsymbol{E} & 0,98384 & -0,49851 & 2,71348 & -2,29778 & 0,21443 & 0,16768 \\ \boldsymbol{I S E} & 1,26239 & -0,83880 & 6,03560 & -6,01910 & 0,47617 & 0,24572\end{array}$

\section{Controlador "industrial" - Servomecanismo}

$\begin{array}{lcccccc} & \mathbf{a} & \mathbf{b} & \mathbf{c} & \mathbf{d} & \mathbf{e} & \mathbf{f} \\ \text { IAE } & 0,81699 & -1,00400 & 1,09112 & -0,22387 & 0,44278 & 0,97186 \\ \boldsymbol{I T A E} & 0,83260 & -0,76070 & 1,00268 & 0,00854 & 0,44243 & 1,11499 \\ \boldsymbol{I S E} & 1,14270 & -0,93650 & 0,99223 & -0,35269 & 0,35308 & 0,78088\end{array}$


El procedimiento de sintonización está basado en el mejor modelo de primer orden más tiempo muerto que se pueda obtener, para lazos de control funcionado como servomecanismos, el criterio de desempeño corresponde a la minimización de alguno de los criterios integrales y el controlador uno de los indicados anteriormente.

Las ecuaciones de sintonización son las mismas que las de Rovira dadas por (12) y los valores de los parámetros $a$ a $f$ se muestran en la Cuadro 2 .

\subsection{Método de Sung, O, Lee, Lee y Yi}

Los métodos de sintonización de controladores que optimizan criterios integrales vistos anteriormente, se basan en un modelo de primer orden más tiempo muerto. (Sung et al., 1996) por su parte basaron su procedimiento de sintonización en un modelo de segundo orden más tiempo muerto para el proceso como el dado por (9b) identificado mediante la realización de una prueba con realimentación por relé seguida por una con control $P$.

El procedimiento está basado en el mejor modelo de segundo orden más tiempo muerto que se pueda obtener, para lazos de control funcionado como servomecanismos, el criterio de desempeño corresponde a la minimización de la ITAE y el controlador un PID-Ideal. El ajuste de las ecuaciones la realizaron para $0,05 \leq t_{m} / \leq 2,0$.

Las ecuaciones de sintonización son:

$$
\begin{aligned}
& K_{c} k_{p}=-0,04+\left[0,333+0,949\left(\frac{t_{m}}{\tau}\right)^{-0.983}\right] \varsigma, \varsigma \leq 0,9 \\
& K_{c} k_{p}=-0,544+0,308\left(\frac{t_{m}}{\tau}\right)+1,408\left(\frac{t_{m}}{\tau}\right)^{-0.832} \varsigma, \varsigma>0,9 \\
& \frac{T_{i}}{\tau}=\left[2,055+0,072\left(\frac{t_{m}}{\tau}\right)\right] \varsigma, \frac{t_{m}}{\tau} \leq 1
\end{aligned}
$$

$$
\begin{aligned}
& \frac{T_{i}}{\tau}=\left[1,768+0,329\left(\frac{t_{m}}{\tau}\right)\right] \varsigma, \frac{t_{m}}{\tau}>1 \\
& \frac{\tau}{T_{d}}=\left[1-\operatorname{EXP}\left(\frac{-\left(t_{m} / \tau\right)^{, 060} \varsigma}{0,870}\right)\right]\left[0,55+1,683\left(\frac{t_{m}}{\tau}\right)^{-1,090}\right]
\end{aligned}
$$

\subsection{Métodos basados en modelo interno}

Las técnicas de control basadas en modelos, incorporan dentro del controlador un modelo del proceso. Este tipo de control es conocido como control con modelo interno, Internal Model Control o IMC, por sus siglas en inglés.

(García et al, 1985) establecieron el concepto de control con modelo interno, en primer lugar, para los sistemas de una entrada y una salida (SISO) y en segundo lugar, para el multivariable (MIMO).

En la Figura 2 se muestra el diagrama de bloques básico del sistema de control basado en modelo, en donde $\breve{G}_{p}(s)$ es un modelo de la planta $G_{p}(s)$ y $G_{C}^{\prime}(s)$ de controlador IMC. Comparando las Figuras 1 y 2, se observa como el controlador $G_{c}(s)$ equivalente está dado por:

$G_{C}(s)=\frac{G_{C}^{\prime}(s)}{1-G_{C}^{\prime}(s) \widetilde{G}_{p}(s)}$

Esta ecuación es la base para la obtención de los controladores del tipo PID-IMC, es decir, controladores PID cuyos parámetros se han determinado aplicando alguna de las técnicas de control con modelo interno.

\subsubsection{Método de Rivera, Morari y Stogestad}

Partiendo de la estructura IMC general, (Rivera et al, 1985), desarrollaron un procedimiento para obtener los controladores y lograr un cierto desempeño deseado. Demostraron que para modelos simples esta estructura conduce a controladores del tipo PID. 
Redefinieron el controlador IMC como:

$G_{C}^{\prime}(s)=\widetilde{G}_{p}^{-1}(s) F(s)$

donde $F(s)$ es un filtro paso bajo, que debe seleccionarse de manera tal para garantizar que la función de transferencia del controlador $I M C$ sea propia. El filtro es de la forma:

$F(s)=\frac{1}{(\lambda s+1)^{n}}$

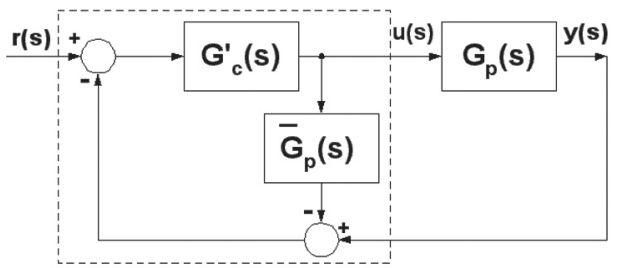

Figura 2. Estructura IMC básica
El Cuadro 3 muestra los parámetros de los controladores PID-IMC obtenidos por Rivera para modelos sin tiempo muerto. Debe tenerse en cuenta que los parámetros del controlador PID son para un PID-Ideal.

Para derivar los parámetros listados en el Cuadro 4 , en el caso de los modelos que incluyen un tiempo muerto, Rivera utilizó una aproximación de Padé de orden cero para el controlador PI y una de primer orden para el PID.

\subsubsection{Método de Brosilow}

En vez de usar las aproximaciones de Padé, empleadas por Rivera, (Brosilow, 1992) utilizó un procedimiento basado en la aproximación de la función de transferencia del controlador por una serie de Maclaurin para derivar las ecuaciones en el cálculo de los parámetros del controlador PID-IMC.

Cuadro 3. Parámetros $P I D-I M C$ de Rivera et al. para plantas sin tiempo muerto

\begin{tabular}{cccccc}
$\begin{array}{c}\text { Modelo } \\
\widetilde{G}_{p}(s)\end{array}$ & $\frac{y(s)}{r(s)}$ & $\begin{array}{c}\text { Controlador } \\
G_{c}(s)\end{array}$ & $\boldsymbol{K}_{c}$ & $\begin{array}{c}\text { Parámetros } \\
T_{i}\end{array}$ & $T_{d}$ \\
\hline$\frac{k_{p}}{\tau s+1}$ & $\frac{1}{\lambda s+1}$ & $\frac{\tau s+1}{\lambda k_{k} s}$ & $\frac{\tau}{\lambda k_{p}}$ & $\tau$ & - \\
$\frac{k_{p}}{\left(\tau_{1} s+1\right)\left(\tau_{2} s+1\right)}$ & $\frac{1}{\lambda s+1}$ & $\frac{\left(\tau_{1} s+1\right)\left(\tau_{2} s+1\right)}{\lambda k_{p} s}$ & $\frac{\tau_{1}+\tau_{2}}{\lambda k_{p}}$ & $\tau_{1}+\tau_{2}$ & $\frac{\tau_{1} \tau_{2}}{\tau_{1}+\tau_{2}}$ \\
$\frac{k_{p}}{\tau^{2} s^{2}+2 \delta s+1}$ & $\frac{1}{\lambda s+1}$ & $\frac{\tau^{2} s^{2}+2 \delta s+1}{\lambda k_{p} s}$ & $\frac{2 \varsigma \tau}{\lambda k_{p}}$ & $2 \varsigma \tau$ & $\frac{\tau}{2 \zeta}$ \\
$\frac{k_{p}}{s}$ & $\frac{1}{\lambda s+1}$ & $\frac{1}{\lambda k_{p}}$ & $\frac{1}{\lambda k_{p}}$ & - & - \\
$\frac{k_{p}}{s(\tau s+1)}$ & $\frac{1}{\lambda s+1}$ & $\frac{2 \lambda s+1}{\lambda^{2} k_{p} s}$ & $\frac{1}{\lambda k_{p}}$ & - & $\tau$
\end{tabular}


Cuadro 4. parámetros PID-IMC de Rivera et al. para plantas con tiempo muerto

\begin{tabular}{|c|c|c|c|c|}
\hline Controlador & $K_{c}$ & $T_{i}$ & $T_{d}$ & $\begin{array}{c}\lambda / t_{m} \text { recomendado } \\
\left(<0.1 \tau / t_{m}\right)\end{array}$ \\
\hline$P I D$ & $\frac{2 \tau+t_{m}}{k_{p}\left(2 \lambda+t_{m}\right)}$ & $\tau+\frac{t_{m}}{2}$ & $\frac{\tau t_{m}}{2 \tau+t_{m}}$ & $>0.8$ \\
\hline$P I$ & $\frac{\tau}{\lambda k_{p}}$ & $\tau$ & - & $>1.7$ \\
\hline $\begin{array}{c}P I \\
\text { método mejorado } \\
(*)\end{array}$ & $\frac{2 \tau / t_{m}}{2 \lambda k_{p}}$ & $\tau+\frac{t_{m}}{2}$ & - & $>1.7$ \\
\hline
\end{tabular}

(*) El tiempo muerto de la planta no fue considerado en el método original para la sintonización del controlador PI, pero si en el método mejorado

Si la planta es de primer orden más tiempo muerto, como la dada por (7), y la función de transferencia de lazo cerrado deseada es:

$G_{l c}(s)=\frac{e^{-t_{m} s}}{\lambda s+1}$

es decir, con ganancia unitaria (error permanente cero) y un parámetro ajustable que permita variar la velocidad de la respuesta del sistema, los parámetros del controlador PID-IMC Ideal deben ser:

$K_{C}=\frac{\tau+t_{m}^{2} /\left[2\left(\lambda+t_{m}\right)\right]}{k_{p}\left(\lambda+t_{m}\right)}=\frac{T_{i}}{k_{p}\left(\lambda+t_{m}\right)}$

$T_{i}=\tau+\frac{t_{m}^{2}}{2\left(\lambda+t_{m}\right)}$

$T_{d}=\frac{t_{m}^{2}}{2\left(\lambda+t_{m}\right)}\left(1-\frac{t_{m}}{3 T_{i}}\right)$

Como se aprecia en (18), todos los parámetros del controlador dependen de la constante de tiempo de lazo cerrado ; si ésta crece, o sea que se desea una respuesta de lazo cerrado más lenta, el controlador PID tiende a convertirse en un $P I$.

\subsubsection{Otros métodos del tipo PID-IMC}

En adición a los métodos basados en los modelos anteriores, se puede mencionar, además, el método de (Chien y Fruehauz, 1990), quienes utilizaron el mismo procedimiento hecho por Rivera, pero para controladores PID-Serie, y el de (Brambilla et al, 1990) quienes lo extienden a plantas de segundo orden más tiempo muerto.

Por su parte (Lee et al 1996) aplica el procedimiento empleado por Brosilow a plantas de segundo orden sobreamortiguadas y sub amortiguadas más tiempo muerto.

\section{PRUEBAS COMPARATIVAS POR SIMULACIÓN}

La bondad de las técnicas de sintonización. descritas anteriormente, se evaluó mediante la simulación digital tanto de las plantas de prueba como de los controladores utilizando el programa VisSim $^{\text {TM }}$ 3.0.

\subsection{Planta de prueba}

Se seleccionó como planta para realizar la comparación de los métodos de sintonización de controladores, un sistema de tercer orden sobre 
amortiguado más tiempo muerto dado por la función de transferencia:

$G_{p}(s)=\frac{e^{-0,1 s}}{(s+1)(0,5 s+1)(0,25 s+1)}$

la cual es una pequeña variante de la planta de prueba Tipo 2. sugerida por (Aström y Hägglund, 2000) dada por

$$
\begin{gathered}
G_{p 2}(s)=\frac{1}{(s+1)(\alpha s+1)\left(\alpha^{2} s+1\right)\left(\alpha^{3} s+1\right)} \\
=0,1 \quad 0,2 \quad 0,5 \quad 1,0
\end{gathered}
$$

Se tomó el valor de $=0,5$ y el polo más rápido de la planta se reemplazó por un tiempo muerto puro de 0,1 unidades de tiempo. Esto último permitió investigar el efecto que el tiempo muerto pudiera tener sobre la respuesta del sistema de control. Se supuso que la unidad de tiempo era el minuto.

\section{2 Índices y criterios de desempeño}

Para evaluar el comportamiento dinámico de los sistemas de control y poder comparar la bondad de los métodos de sintonización estudiados se tomaron los siguientes índices:

\section{- Error máximo}

Se definió como uno de los criterios de desempeño el Porcentaje de Error Máximo (PEM) dado por

$P E M=\frac{100\left|y(t)_{\max }-r(t)\right|}{r(t)}$

en el caso de los sistemas operando como servomecanismo y como

$P E M=100\left|y(t)_{\max }\right|$

en el caso de los sistemas operando como reguladores.

\section{- Tiempo de asentamiento}

Se seleccionó como otro de los criterios de desempeño el Tiempo de Asentamiento $\left(\mathrm{Ta}_{2}\right)$. Tiempo requerido por el sistema para que el error entrara en una banda del $\pm 2 \%$ del valor deseado

$T a_{2}=t_{a} \ni|y(t)-r(t)| \leq 0,02 r(t) \forall t \geq t_{a}$

\section{- Integral del error absoluto}

Con base al error se definió el índice de desempeño

$I A E=\int_{0}^{\infty}|y(t)-r(t)| d t$

El IAE da el área bajo la curva del error la cual representa la cantidad de material fuera de especificaciones, energía perdida u otra característica indeseada. Si el IAE $\rightarrow 0$ entonces $y(t) \quad r(t) \quad t$ y el control fuese perfecto.

Aunque la magnitud del IAE es una indicación de la bondad del método de sintonización respecto al error, es más útil para efectos de comparación respecto a otros métodos de sintonización, su valor relativo respecto al valor del IAE que se obtendría si los parámetros del controlador fueran óptimos respecto a ese criterio. Se definió entonces un Índice de Desempeño en el Error $(I D E)$ de un controlador sintonizado con un conjunto de parámetros que produce un valor determinado IAE como

$I D E=\frac{I A E_{o p t}}{I A E}$

donde $I A E_{o p t}$ es el valor del IAE para el sistema con el controlador "óptimo" respecto a este criterio.

\section{- Índices de robustez}

La sola medición del desempeño del lazo de control no puede constituirse en la forma de evaluar la bondad de los métodos de sintonización 
de controladores. El cálculo de los parámetros del controlador se basa en la obtención de información del proceso a controlar en un punto de operación, usualmente mediante la identificación de un modelo de primer o segundo orden más tiempo muerto. Los parámetros de esos modelos (ganancia, constantes de tiempo y tiempo muerto aparente) dependen del punto en el cual se realizó la identificación. Aunque esta se realice en el punto de operación más probable del sistema, la naturaleza no lineal intrínseca en los procesos, hace que los valores de estos parámetros cambien debido al efecto de las perturbaciones o de cambios en el valor deseado.

Una característica deseada para los métodos de sintonización de controladores, es que el sistema de control conserve una operación estable ante estos cambios en los parámetros de la planta, siendo la variación requerida de los mismos para llevar el sistema al límite de la estabilidad una indicación de esta habilidad, la cual se denomina robustez.

Dos de los parámetros de la planta que pueden sufrir variaciones son la ganancia y el tiempo muerto. Se definieron entonces los siguientes Índices de Robustez $\left(I R k_{p}, I R t_{m}\right)$ respecto a la variación de estos parámetros como:

$I R k_{p}=\frac{k_{p u}}{k_{p a}}-1 ; \quad t_{m}=t_{m a}$

$I R t_{m}=\frac{t_{m u}}{t_{m a}}-1 ; k_{p}=k_{p a}$

en donde los parámetros con subíndice " $a$ " denotan los valores utilizados en cálculo de los parámetros del controlador y los con subíndice " $u$ " los valores que llevan el sistema al límite de la estabilidad.

Los índices de robustez (26) y (27) son mediciones independientes de las variaciones requeridas en la ganancia y en el tiempo muerto del sistema para que el sistema se inestabilice, esto es considerando cada uno por separado, pero si la variación es simultánea el sistema se puede volver inestable mucho antes de que los parámetros alcancen los valores establecidos por los índices de robustez.

Se definió entonces un Índice de Robustez Absoluta (IRA) dado por la siguiente expresión

$I R A=\min \left(I R k_{p}, I R t_{m}, 0.5\left[I R k_{p}^{2}+I R t_{m}^{2}\right]^{1 / 2}\right)$

\section{3 Índice de desempeño de criterio múltiple}

En el punto 4.2 anterior se establecieron varios índices o criterios de desempeño a ser utilizados para medir la bondad relativa de los diferentes métodos de sintonización a comparar.

Se establecerá aquí entonces un Índice de Desempeño Absoluto (IDA) multicriterio que incluye y pondera de alguna manera los criterios de desempeño ya indicados el cual se definió como:

$I D A_{i}=\frac{\left[\frac{20}{P E M_{i}}+\frac{T a_{2 \mathrm{max}}}{T a_{2}}+1.5 I D E_{i}+\frac{I R A_{i}}{2 \sqrt{2}}\right]}{4}$

para $P E M \geq 20$

$I D A_{i}=\frac{\left[1+\frac{T a_{2 \max }}{T a_{2}}+1.5 I D E_{i}+\frac{I R A_{i}}{2 \sqrt{2}}\right]}{4}$

para $P E M \leq 20$

Los coeficientes de ponderación incluidos en el criterio IDA se seleccionaron de manera de que el peso relativo de cada criterio sea similar, entonces:

- Se penalizaron las respuestas que tenían más de un $20 \%$ de error máximo o sea valores de PEM superiores a 20.

- Como el tiempo de asentamiento del sistema $\mathrm{Ta}_{2}$ no depende solamente de los parámetros del controlador, sino que también depende de las características dinámicas de la planta, se 
le asignó un peso unitario dentro del criterio al tiempo de asentamiento del método que produjera la respuesta más lenta, $T a_{2 \max }$, teniendo los demás métodos de sintonización factores superiores a 1 por este concepto.

- Ya que el índice de desempeño en el error $I D E$ puede tener un valor máximo de 1 , se consideró entonces que el método de sintonización debía producir valores del $I A E$ que fueran a lo más un $50 \%$ mayores que el óptimo para que este componente del criterio tuviera valores superiores a 1 .

- Siendo que usualmente se requiere que los indices de robustez IRk $k_{p}$ y $I R t_{m}$ sean superiores a 1 , esto es que el sistema permanezca estable ante variaciones independientes de hasta un $100 \%$ en la ganancia o el tiempo muerto del proceso, el $I R A$ tendría un valor cercano a $0,5 \sqrt{ } 2$ como mínimo aceptable, y se consideró deseable entonces que el IRA fuera superior a $2 \sqrt{ } 2$.

\subsection{Identificación de la planta de prueba}

Se obtuvo la curva de reacción de la planta de prueba (19) y se determinaron los valores necesarios para la identificación de modelos de primer y segundo orden más tiempo muerto mediante varios métodos de identificación.

Los parámetros de los modelos se muestran en los Cuadros 5 y 6 los cuales incluyen el valor de la Integral del Error de Predicción Absoluto (IAEP) de cada uno de ellos. La bondad relativa de los modelos identificados concuerda con los resultados obtenidos con otras plantas de prueba (Alfaro, 2001a).

Se utilizaron los parámetros del modelo de primer orden más tiempo muerto identificado por el método de Ho, con la excepción de en el método de sintonización de Sung el cual requiere de un modelo de segundo orden más tiempo muerto.

Cuadro 5. Modelos de primer orden más tiempo muerto

Método
Alfaro
Ho et al,
Miller
Smith
Vitecková et al.
Ziegler y Nichols

$\begin{array}{cccc}\boldsymbol{k}_{\boldsymbol{p}} & \boldsymbol{t}_{\boldsymbol{m}} & \boldsymbol{I A E P} \\ 1 & 1,23 & 0,68 & 0,095 \\ 1 & 1,13 & 0,77 & 0,088 \\ 1 & 1,47 & 0,49 & 0,190 \\ 1 & 1,29 & 0,67 & 0,114 \\ 1 & 1,22 & 0,71 & 0,093 \\ 1 & 2,17 & 0,49 & 0,695\end{array}$

Cuadro 6. Modelos de segundo orden más tiempo muerto

\begin{tabular}{|c|c|c|c|c|c|c|c|}
\hline Método & $k_{p}$ & 1 & 2 & $t_{m}$ & & $n$ & $I A E P$ \\
\hline Stark & 1 & - & - & 0,242 & 0,930 & 1,184 & 0,049 \\
\hline Vitecková & 1 & 0,78 & 0,78 & 0,282 & - & - & 0,026 \\
\hline
\end{tabular}

Cuadro 7. Parámetros óptimos del controlador PID

$\begin{array}{lcccccc}\text { Sistema control } & \boldsymbol{K}_{\boldsymbol{c}} & \boldsymbol{T}_{\boldsymbol{i}} & \boldsymbol{T}_{\boldsymbol{d}} & \boldsymbol{I A E} & \boldsymbol{T a}_{\boldsymbol{2}} & \boldsymbol{P E M} \\ \text { Servomecanismo } & 3,061 & 1,610 & 0,483 & 0,6255 & 2,488 & 9,55 \\ \text { Regulador } & 7,112 & 0,598 & 0,468 & 0,1324 & 3,269 & 12,78\end{array}$




\subsection{Parámetros óptimos del controlador}

Para poder establecer el Índice de Desempeño en el Error IDE de cada uno de los métodos de sintonización, tal como se indicó en 4.2 y 4.3 se obtuvieron los conjuntos de parámetros $\left(K_{c}, T_{i}\right.$, $T_{d}$ ) que minimizan el criterio IAE para el sistema operando tanto como servomecanismo como como regulador.

Los resultados de la optimización se muestran en el Cuadro 7.

\subsection{Parámetros de los controladores}

Los parámetros calculados con los métodos de sintonización descritos en 3.0 aplicables a un controlador PID-Ideal se muestran en el Cuadro 8. y los correspondientes a controladores PIDSerie o PID-Industrial en los Cuadros 9 y 10 respectivamente.

\subsection{Resultados de la simulación}

Los controladores $P I D$ fueron sintonizados con los parámetros calculados en 4.6 y se obtuvo la respuesta del sistema de control a un cambio escalón en el valor deseado, determinándose los valores de las variables necesarias para el cálculo de los índices de desempeño, establecidos en 4.2 y 4.3 .

Los resultados obtenidos se muestran en los Cuadros 11, 12 y 13.

Cuadro 8. Parámetros para el controlador PID-Ideal

Método
Rovira et al. - IAE $($ RIAE $)$
Rovira et al. - ITAE $($ RITAE)
Sung et al. $($ SungS $)$
Rivera et al. $(=0,25 \mathrm{t})$
Rivera et al. $(=0,50 \mathrm{t})$
Rivera et al. $(=0,75 \mathrm{t})$
Rivera et al. $(=1,00 \mathrm{t})$
Rivera et al. $(=1,25 \mathrm{t})$
Brosilow et al. $(=0,25 \mathrm{t})$
Brosilow et al. $(=0,50 \mathrm{t})$
Brosilow et al. $(=0,75 \mathrm{t})$
Brosilow et al. $=1,00 \mathrm{t})$
Brosilow et al. $(=1,25 \mathrm{t})$

$\begin{array}{ccc}\boldsymbol{K}_{\boldsymbol{c}} & \boldsymbol{T}_{\boldsymbol{i}} & \boldsymbol{T}_{\boldsymbol{d}} \\ 1,516 & 1,735 & 0,277 \\ 1,337 & 1,623 & 0,244 \\ 3,250 & 1,631 & 0,480 \\ 2,386 & 1,515 & 0,287 \\ 1,712 & 1,515 & 0,287 \\ 1,335 & 1,515 & 0,287 \\ 1,094 & 1,515 & 0,287 \\ 0,927 & 1,515 & 0,287 \\ 1,392 & 1,420 & 0,238 \\ 1,073 & 1,363 & 0,189 \\ 0,872 & 1,325 & 0,157 \\ 0,733 & 1,297 & 0,134 \\ 0,363 & 1,277 & 0,117\end{array}$

Cuadro 9. Parámetros para el controlador PID-Serie

$\begin{array}{lccc}\text { Método } & \boldsymbol{K}_{\boldsymbol{c}} & \boldsymbol{T}_{\boldsymbol{i}} & \boldsymbol{T}_{\boldsymbol{d}} \\ \text { Kaya y Shieb - IAE }(\text { KSIAEs }) & 0,970 & 1,072 & 0,379 \\ \text { Kaya y Shieb - ITAE }(\text { KSITAEs }) & 1,535 & 1,111 & 0,329 \\ \text { Kaya y Shieb - ISE (KSISEs) } & 1,069 & 1,127 & 0,443\end{array}$


Las curvas de respuesta de los diferentes métodos de sintonización se muestran en las Figuras 3, 4 y 5 .

\subsection{Análisis de los resultados}

En 4.3 se estableció un Índice de Desempeño Absoluto (IDA), incorporando en un solo factor de evaluación, diferentes características de la respuesta temporal del sistema posibles de medir, tales como el error máximo, el tiempo de asentamiento y la integral del valor absoluto del error, así como la robustez del conjunto de parámetros calculados.

Como se puede apreciar en el Cuadro 11, a medida que se incrementa el valor del parámetro de sintonización en los métodos del tipo $I M C$, aumenta rápidamente la robustez del método, mucho más allá de los valores mínimos normalmente deseables, debido a que la ganancia requerida del controlador disminuye apreciablemente.

Así, su índice de robustez absoluta IRA tiene una gran incidencia sobre el valor obtenido para su índice de desempeño absoluto IDA. Por esta razón, se incluyó en los Cuadros 11, 12 y 13 un Índice de Desempeño Absoluto modificado $I D A m$, el cual excluye de la ponderación la robustez del método. Como se puede apreciar, para los métodos con IRA superior a 6, su IDAm es menor comparado con el valor de su $I D A$.

En el Cuadro 13 se muestra los métodos estudiados y ordenados según el valor del IDAm obtenido con cada uno de ellos y la posición relativa, según cada criterio individual.

Cuadro 10. Parámetros para el controlador PID-Industrial

$\begin{array}{lccc}\text { Método } & \boldsymbol{K}_{\boldsymbol{c}} & \boldsymbol{T}_{\boldsymbol{i}} & \boldsymbol{T}_{\boldsymbol{d}} \\ \text { Kaya y Shieb - IAE }(\text { KSIAEs }) & 1,200 & 1,204 & 0,500 \\ \text { Kaya y Shieb - ITAE }(\text { KSITAEs }) & 1,115 & 1,120 & 0,326 \\ \text { Kaya y Shieb - ISE }(\text { KSISEs) } & 1,637 & 1,503 & 0,296\end{array}$

Cuadro 11. Índices de desempeño, controlador PID-Ideal

\begin{tabular}{|c|c|c|c|c|c|c|c|c|c|}
\hline Método & PEM & $T a_{2}$ & $I A E$ & $I D E$ & $I R k_{p}$ & $I R t_{m}$ & $I R A$ & $I D A$ & $I D A m$ \\
\hline RIAE & $-(*)$ & 4,95 & 1,144 & 0,547 & 7,20 & 13,00 & 7,20 & 1,63 & 1,33 \\
\hline RITAE & - & 4,73 & 1,214 & 0,515 & 8,25 & 14,15 & 8,19 & 1,74 & 1,35 \\
\hline SungS & 12,33 & 2,45 & 0,627 & 0,998 & 1,95 & 4,20 & 1,95 & 1,89 & 2,29 \\
\hline Rivera $(=0,25 \quad)$ & 11,46 & 3,83 & 0,824 & 0,759 & 3,90 & 6,30 & 3,71 & 1,56 & 1,65 \\
\hline Rivera $(=\mathbf{0 , 5 0})$ & 2,30 & 2,49 & 0,919 & 0,681 & 5,85 & 9,90 & 5,75 & 2,10 & 2,11 \\
\hline Rivera (= 0,75 ) & - & 2,84 & 1,135 & 0,551 & 7,80 & 13,65 & 7,80 & 2,09 & 1,87 \\
\hline Rivera (= 1,00 ) & - & 4,49 & 1,384 & 0,452 & 9,70 & 17,60 & 9,70 & 1,88 & 1,36 \\
\hline Rivera (= 1,25 ) & - & 5,54 & 1,634 & 0,383 & 11,65 & 21,50 & 11,65 & 1,91 & 1,17 \\
\hline Brosilow $(=0,25$ & 2,81 & 3,21 & 1,077 & 0,581 & 7,15 & 11,60 & 6,81 & 1,91 & 1,74 \\
\hline Brosilow $(=\mathbf{0 , 5 0}$ & 1,25 & 2,68 & 1,295 & 0,483 & 8,10 & 14,85 & 8,10 & 2,15 & 1,91 \\
\hline Brosilow $(=0,75$ & - & 3,44 & 1,519 & 0,412 & 6,75 & 18,25 & 8,85 & 1,96 & 1,58 \\
\hline Brosilow $(=1,00$ & - & 4,50 & 1,769 & 0,354 & 9,25 & 22,00 & 9,25 & 1,80 & 1,31 \\
\hline Brosilow $(=1,25$ & - & 12,01 & 3,506 & 0178 & 17,80 & 49,50 & 17,80 & 2,11 & 0,72 \\
\hline
\end{tabular}


Cuadro 12. Índices de desempeño, controlador PID-Serie

$\begin{array}{lccccccccc}\text { Método } & \boldsymbol{P E M} & \boldsymbol{T a}_{\boldsymbol{2}} & \boldsymbol{I A E} & \boldsymbol{I D E} & \boldsymbol{I R}_{\boldsymbol{p}} & \boldsymbol{I R t}_{\boldsymbol{m}} & \boldsymbol{I R A} & \boldsymbol{I D A} & \boldsymbol{I D A m} \\ \text { KSIAEs } & 1,52 & 2,38 & 1,141 & 0,548 & 7,90 & 13,28 & 7,73 & \mathbf{2 , 2 7} & \mathbf{2 , 1 1} \\ \text { KSITAEs } & 11,74 & 4,16 & 0,981 & 0,638 & 4,80 & 7,50 & 4,45 & \mathbf{1 , 5 3} & \mathbf{1 , 5 1} \\ \text { KSISEs } & - & 2,35 & 1,054 & 0,593 & 6,70 & 12,50 & 6,70 & \mathbf{2 , 2 1} & \mathbf{2 , 1 5}\end{array}$

Cuadro 13. Índices de desempeño, controlador PID-Industrial

$\begin{array}{lccccccccc}\text { Método } & \boldsymbol{P E M} & \boldsymbol{T a}_{\boldsymbol{2}} & \boldsymbol{I A E} & \boldsymbol{I D E} & \boldsymbol{I R}_{\boldsymbol{p}} & \boldsymbol{I R t}_{\boldsymbol{m}} & \boldsymbol{I R A} & \boldsymbol{I D A} & \boldsymbol{I D A} \boldsymbol{m} \\ \text { KSIAEs } & - & 4,28 & 1,453 & 0,430 & 5,50 & 11,60 & 5,50 & \mathbf{1 , 5 3} & \mathbf{1 , 3 9} \\ \text { KSITAEs } & 3,03 & 3,75 & 1,368 & 0,457 & 7,10 & 11,50 & 6,76 & \mathbf{1 , 7 4} & \mathbf{1 , 5 2} \\ \text { KSISEs } & 2,23 & 4,96 & 1,209 & 0,518 & 5,70 & 8,75 & 5,22 & \mathbf{1 , 4 5} & \mathbf{1 , 3 1}\end{array}$

Cuadro 14. Posición relativa de los métodos según el IDAm

\begin{tabular}{|c|c|c|c|c|c|c|}
\hline Método & IDAm & $I D A$ & PEM & $\mathrm{Ta}_{2}$ & $I D E$ & IRA \\
\hline Sungs & 2,29 & 1,89 & $(9)^{*}$ & (3) & (1) & (19) \\
\hline KSISEs (Serie) & 2,15 & 2,21 & $-* *$ & (1) & (5) & (13) \\
\hline KSIAEs (Serie) & 2,11 & 2,27 & (2) & (2) & (8) & (9) \\
\hline Rivera ( =0,50 ) & 2,11 & 2,10 & (4) & (4) & (3) & (14) \\
\hline Brosilow ( =0,50 ) & 1,91 & 2,15 & (1) & (5) & (12) & (7) \\
\hline Rivera ( =0,75 ) & 1,87 & 2,09 & - & (6) & (7) & (8) \\
\hline Brosilow ( =0,25 ) & 1,74 & 1,91 & (5) & (7) & (6) & (11) \\
\hline Rivera ( =0,25 ) & 1,65 & 1,56 & (7) & (10) & (2) & (18) \\
\hline Brosilow ( $=0,75$ ) & 1,58 & 1,96 & - & (8) & (16) & (5) \\
\hline KSITAEs (Industrial) & 1,52 & 1,74 & (6) & (9) & (13) & (12) \\
\hline KSITAEs (Serie) & 1,51 & 1,53 & (8) & (11) & (4) & (17) \\
\hline KSIAEs (Industrial) & 1,39 & 1,53 & - & (12) & (15) & (15) \\
\hline Rivera ( =1,00 ) & 1,36 & 1,88 & - & (13) & (14) & (3) \\
\hline RITAE & 1,35 & 1,74 & - & (16) & (11) & (6) \\
\hline RIAE & 1,33 & 1,63 & - & (15) & (9) & (10) \\
\hline Brosilow ( =1,00 ) & 1,31 & 1,88 & - & (14) & (18) & (4) \\
\hline KSISEs (Industrial) & 1,31 & 1,45 & (3) & (17) & (10) & (16) \\
\hline Rivera ( =1,25 ) & 1,17 & 1,91 & - & (18) & (17) & (2) \\
\hline Brosilow ( =1,25 ) & 0,72 & 2,11 & - & (19) & (19) & (1) \\
\hline
\end{tabular}

(*) los números en paréntesis indican la posición relativa del método respecto a cada uno de los criterios $(* *)$ respuesta sobreamortiguada 
Si se considera cada criterio de forma individual, se nota que el criterio del PEM menor al $20 \%$ lo cumplen todos los métodos, siendo el sobrepaso más alto el del método de Sung con solo un $12,33 \%$. Todas las respuestas están bastante amortiguadas.

La respuesta con el menor tiempo de asentamiento, $T_{a 2}$, se logra con los métodos de Kaya y Sheib para controladores PID-Serie y criterios ISE e $I A E$, seguidas muy de cerca por la respuesta del método de Sung.

El criterio establecido, según el cual el IAE sea un $50 \%$ mayor que el mínimo, es decir, un $I D E$ mayor a 0,67 , solo lo cumple el método de Sung y el de Rivera para valores bajos de $(=0,25$ y 0,5$)$. En el caso de los métodos tipo IMC, y en especial en el de Brosilow, se ve claramente como al aumentar el valor de, su respuesta se hace más lenta y su $I A E$ crece haciéndola inaceptable.

El criterio de que el IRA fuera superior a $2 \sqrt{ } 2$ lo cumplen todos excepto el de Sung, el cual resultó ser el menos robusto. Sin embargo, el valor de su $I R k_{p}$ es $1,95 \mathrm{y}$ el de su $I R t_{m} 4,20$ que excede el mínimo aceptable de 1 . Se puede observar, además, como todos los métodos dan sistemas más robustos en cambios en el tiempo muerto que en cambios en la ganancia del proceso.

Al considerar los criterios en forma simultánea, se encuentra que no hay ningún método que los cumpla todos. Aunque el método de Sung se encuentra en primer lugar del $I D A_{m}$, cae al décimo si se considera el IRA.
Los resultados anteriores indican que habrá que establecer un conciliación entre los diferentes criterios de desempeño en el momento de seleccionar el método de sintonización.

\section{CONCLUSIONES}

De los resultados obtenidos con los métodos de sintonización para servomecanismos, se puede concluir que si lo que se desea es una respuesta rápida con un sobrepaso máximo bajo, el método de Sung sería el adecuado. Las curvas de respuesta en la Figura 3 muestran claramente la bondad de este ajuste el cual prácticamente coincide con el óptimo, bajo el criterio IAE.

Los métodos de Kaya y Sheib y el de Rovira dan respuestas iniciales más lentas como se muestra en la Figura 4.

Los métodos basados en modelo, Rivera o Brosilow, permiten mediante la variación del parámetro de sintonización de lazo cerrado , obtener respuestas prácticamente sin sobrepaso, pero lentas para valores de altos, o rápidas pero con sobrepaso si el valor del parámetro de sintonización se diminuye. Los resultados experimentales mostrados en la Figura 5 permiten recomendar seleccionar la constante de tiempo de lazo cerrado con base en la constante de tiempo de la planta como $0,10 \leq \leq 0,50$.

Si una robustez alta no es indispensable, el método de Sung, en definitiva, sería la mejor opción para sintonizar el controlador PID que opere como servomecanismo. 


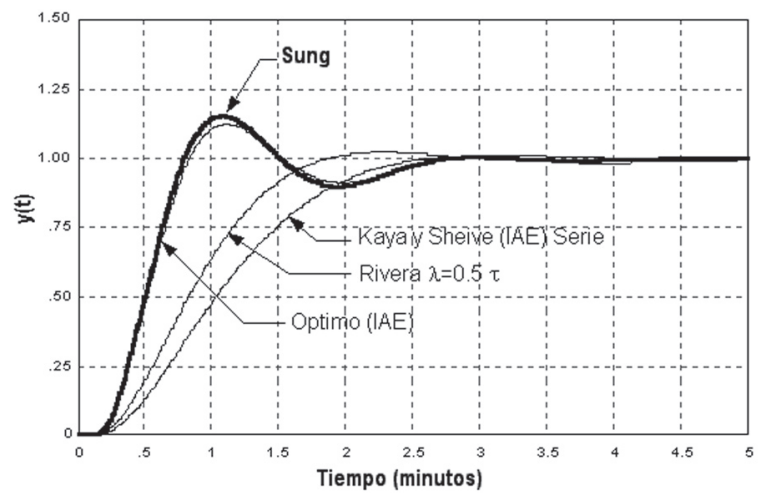

Figura 3. Respuesta a un cambio en el valor deseado

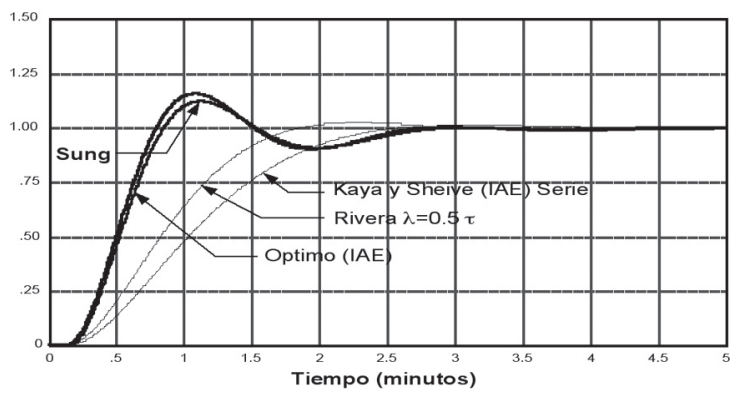

Figura 4. Respuesta a un cambio en el valor deseado

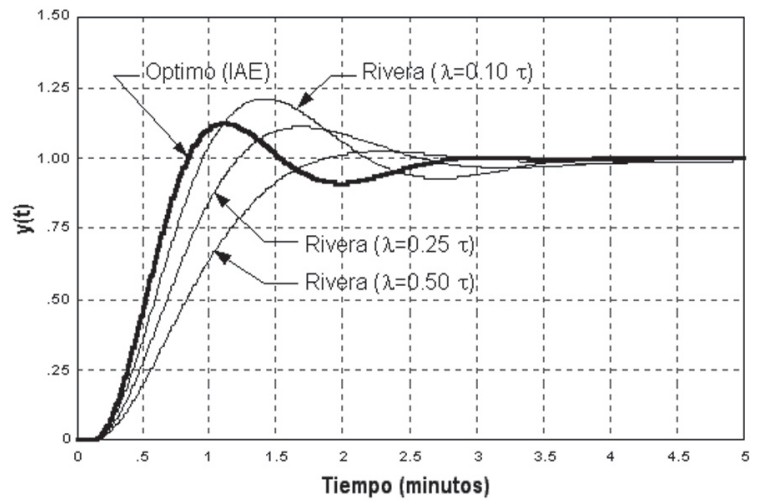

Figura 5. Respuesta a un cambio en el valor deseado 


\section{SIMBOLOGÍA}

$G_{p}(s) \quad$ función de transferencia (modelo)

$G_{c}(s)$ función de transferencia (controlador)

$k_{p} \quad$ ganancia estática

,$\quad$, 2 constantes de tiempo

$t_{m} \quad$ tiempo muerto aparente

$n \quad$ frecuencia natural

razón de amortiguamiento

$s \quad$ variable compleja

Kc ganancia del controlador

$T_{i} \quad$ tiempo integral

$T_{d} \quad$ tiempo derivativo

$K_{c u}, T_{u}$ parámetros últimos

$r(t) \quad$ valor deseado

$y(t) \quad$ variable controlada (señal realimentada)

$z(t) \quad$ perturbación

\section{BIBLIOGRAFÍA}

Alfaro, V. M. (1993). ¿Son todos los controladores PID iguales? Ingeniería, 1(3), 11-9.

Alfaro, V. M. (2001a). Identificación de procesos sobreamortiguados utilizando técnicas de lazo abierto. Ingeniería, 1-2(11), 11-25.

Alfaro, V. M. (2001b). Identificación de procesos sobreamortiguados utilizando técnicas de lazo cerrado. Ingeniería, 1-2(11), 27-40.

Aström, K. J. \& Hägglund, T. (2000). Bench-mark systems for PID control, IFAC Workshop on Digital Control: Past, Present and Future of PID Control, Terrasa, España.

Brambilla, A., Chen, S. \& Scall, C. (1990). Robust tuning of conventional controllers, Hydrocarbon Processing, November, 69(11).

Brosilow C. (1992). PI/PID Controller parameters from IMC design. Department of Chemical Engineering, Case West Reserve University.

Chien, I. L. \& Fruehauf, P. S. (1990). Considerer IMC tuning to improve controller performance, Chemical Engineering Progress. October.

García, C.E. y M. Morari, M. (1982). Internal model control. 1. A Unifying Review and Some New Results, Ind. Eng. Chem. Process Des. Dev. (EUA), 21(2), 308-323.

García, C. E. \& Morari, M. (1982). Internal model control. 2. Design Procedure for Multivariable Systems, Ind. Eng. Chem. Process Des. Dev. 24(2), 472-484.

García, C. E. \& Morari, M. (1985). Internal model control. 3. Multivariable Control Law Computation and Tuning Guide-lines, Ind. Eng. Chem. Process Des. Dev. 24(2), 484 - 494.

Kaya, A. \& Sheib, T. J. (1988). Tuning of PID controllers of different structures. Control Engineering, December 62-65. 1988.

Lee, T., Park, S., Lee, M. \& Brosilow, C. (1996). PID Controller tuning to obtain desired closed-loop response for single input, single output systems. Dept. of Chemical Engineering, Korean Advanced Institute of Science and Technology Taejon, Korea.

O'Dwyer A. (2000a). A summary of PI and PID controller tuning rules for processes with time delay. Part 1: PI Controller Tuning Rules, IFAC Workshop on Digital Control: Past, Present and Future of PID Control, Terrasa, España.

O'Dwyer A. (2000b). A summary of PI and PID controller tuning rules for processes with time delay. Part 2: PID Controller Tuning Rules, IFAC Workshop on Digital Control: Past, Present and Future of PID Control, Terrasa, España.

Rivera, D. E., Morari, M. \& Stogestad, S. (1985). Internal model control. 4. PID Controller Design. Ind. Eng. Chem. Process Des. Dev. 25(1), 252-265. 
Rovira, A., Murrill, P. W. \& Smith, C. L. (1969). Tuning controllers for setpoint changes, Instrumentation \& Control Systems, December 67-69.

Sung, S. W., O, J., Lee, I.B., Lee, J., Yy, S. H.(1996). Automatic tuning of PID controller using second-order plus time delay model, Journal of Chemical Engineering of Japan, 29(6), 990-999.

Visual Solutions - VisSim User's Guide Version 3, Westford, Ma., EUA, Visual Solutions, 1999.

\section{SOBRE EL AUTOR}

\section{Víctor M. Alfaro Ruiz}

Profesor Asociado, Departamento de Automática, Escuela de Ingeniería Eléctrica, Universidad de Costa Rica

Apartado postal 2-10, 2060 UCR, San José, Costa Rica

Teléfono: 207-4472, Fax. 207-4139

Correo electrónico: valfaro@eie.ucr.ac.cr

El presente trabajo fue realizado como parte del proyecto $N^{o}$ 731-A0-169 inscrito en la Vicerrectoría de Investigación de la Universidad de Costa Rica. 